\section{A Comparison of Biofeedback and Occlusal Adjustment on Bruxism}

by

DR. B. J. R. KARDACHI*, $\dagger$

Dr. J. O. BAILEY $\ddagger$

Dr. M. M. Ash§

RECORDINGS of electromyographic (EMG) activity during nocturnal sleep in the home environment are a necessary aid in obtaining an accurate indication of parafunctional activity during sleep and to develop methods aimed at its control. There are very few reports in the literature on EMG related nighttime bruxing activity, mainly due to a lack of suitable recording equipment. Also from an ethical standpoint it is important that parafunctional activity be controlled with an occlusal adjustment, splint, or other forms of therapy rather than be studied because of deleterious effects that may arise. The development of small, portable EMG amplifiers usable during sleep now has made it possible to measure muscle activity and to study the effects of various treatment methods on it. ${ }^{1}$

The only previous electromyographic study to observe the effects of an occlusal adjustment on bruxism was the study by Ramfjord in $1961 .^{2}$ This was performed on wakeful subjects under laboratory conditions, a totally different environment from the subject sleeping at home. Other claims of elimination or control of bruxism by occlusal therapy are based on clinical impressions or patient awareness. ${ }^{3}$

Recently portable EMG equipment has been used to demonstrate the effectiveness of biofeedback therapy in reducing EMG activity during sleep. ${ }^{4}$ The reduction appears to be only temporary in that the muscle activity returns to its initial level on cessation of the feedback treatments. ${ }^{5}$

The purposes of this study were to: (1) determine the effects of an occlusal adjustment on muscle activity in subjects who brux; and (2) compare these effects to those of biofeedback.

\footnotetext{
* Visiting Professor, Department of Occlusion, Dental School, University of Michigan, Ann Arbor, Mich.

$\dagger$ Present Address: Department of Dental Health, Dental School, University of Adelaide, Adelaide, South Australia.

$\ddagger$ Department of Occlusion, Dental School, University of Michigan, Ann Arbor, Mich.

$\S$ Department of Occlusion, Dental School, University of Michigan, Ann Arbor, Mich.
}

\section{Materials and Methods}

Twenty subjects were used for this investigation, divided into two experimental groups of four and three control groups of four. Of the total number, there were 8 females and 12 males, with ages ranging from 18 to 39 years.

The EMG activity during sleep was measured with portable equipment described previously. ${ }^{6}$ The EMG amplifier was designed with adjustable sensitivity so that the EMG activity of swallowing and other functional movements was not recorded; generally the amplifier operated at a threshold of approximately 100 mv. Activity from within the masseter and anterior temporalis muscles was recorded with surface electrodes on one side of the face. The equipment was operated easily by the subject at home.

With the occlusal adjustment group (three males and one female) muscle activity was recorded for 7 nights in an attempt to establish a baseline of (EMG) activity. An occlusal adjustment was then performed according to the concepts of Ramfjord and $\mathrm{Ash}^{7}$ and refined 1 week later as necessary. The EMG activity was collected on a simple portable cassette recorder $\|$ during this entire period and analyzed on a totalizing electronic counter so that a quantitative assessment of EMG activity before and after the adjustment could be made.

On the control occlusal adjustment group ( 1 male, 3 females), a mock adjustment was performed, that is nonopposing, nonsupporting tooth surfaces were ground; the patient's movements and mandibular positions were never guided by the operator. EMG activity was measured for 7 days before and after this "adjustment".

The subjects of the biofeedback treatment group (two males, two females) were recorded for 1 week to establish a baseline of activity; feedback was applied for a similar period, and the recordings continued for a further week.

In this study an attempt was also made to apply some form of control feedback, so that the effect of the application of noise not related to EMG activity could be assessed. The timing apparatus of a common clock was used with a probe passing over active contacts on a printed circuit board at irregular time intervals. The sound produced was a constant tone of varying duration (3-7 seconds) and it occurred from six to eight times each hour. The time interval between sounds varied from 5 minutes to 25 minutes. The EMG activity was recorded as for the biofeedback group.

The fifth group of subjects was diagnosed clinically as nonbruxers by lack of evidence of faceting or knowledge of parafunctional activity. EMG activity was measured on these subjects for 7 nights.

\| Sharp RD-472E. 
The biofeedback and occlusal adjustment experimental groups were recorded again for several nights after 3 months so that any long term effects of either form of therapy could be determined.

\section{Results}

The effect of the occlusal adjustment on nocturnal EMG activity varied. There was an immediate increase in the mean activity in two subjects (subject A $26.1 \%$, subject B 5.3\%) (Fig. 1). After 3 months, subject A demonstrated a reduction of $49.5 \%$ in mean activity and some decrease in the range of activity while activity for subject $B$ had returned to the initial level. In the other two subjects there was an initial decrease in mean activity following the adjustment (subject $\mathrm{C} 17.3 \%$, subject D 22.7\%) (Fig. 2). At the 3-month recording session the activity of subject $C$ had returned to the initial level while that for subject $\mathrm{D}$ was $15 \%$ less than the preadjustment level.

In the mock adjustment group, there was a slight decrease in the mean EMG activity in all four subjects following the treatment procedure (Fig. 3).

Biofeedback resulted in a reduction of approximately $70 \%$ in all four subjects while on the feedback (subject A $70.5 \%$, subject B $61.2 \%$, subject C $80.5 \%$, and subject D 72.8\%) (Figs. 4 and 5). A return to approximately the original level was observed in three patients on cessation of the input, while in subject $C$ the activity remained low, but had returned to the original level after 3 months. Subject B showed a reduction of $50 \%$ at the 3-month interval. The range of activity between nights was reduced in all four subjects while on biofeedback therapy, but increased again following treatment.

The control feedback group demonstrated no substantial change in mean EMG activity during the experimental period, there being a slight increase in activity in two subjects and a slight decrease in the other two subjects (Fig. 6).

The group assessed clinically as nonbruxers demonstrated very little EMG activity during the recording period. Two subjects recorded zero activity, while in the other two, the level of activity was much less than any of the experimental subjects.

\section{Discussion}

The results of this pilot investigation into the effects of methods to control EMG activity of the masticatory muscles during sleep show the need for a long term study in a large population before definitive conclusions can be made. A long term study, however, will not be easy as this program has shown that there is some difficulty in acquiring subjects to use this type of equipment for an extended period of time. The recording periods chosen arbitrarily here of 1 week for each sequence are probably minimal time periods and longer

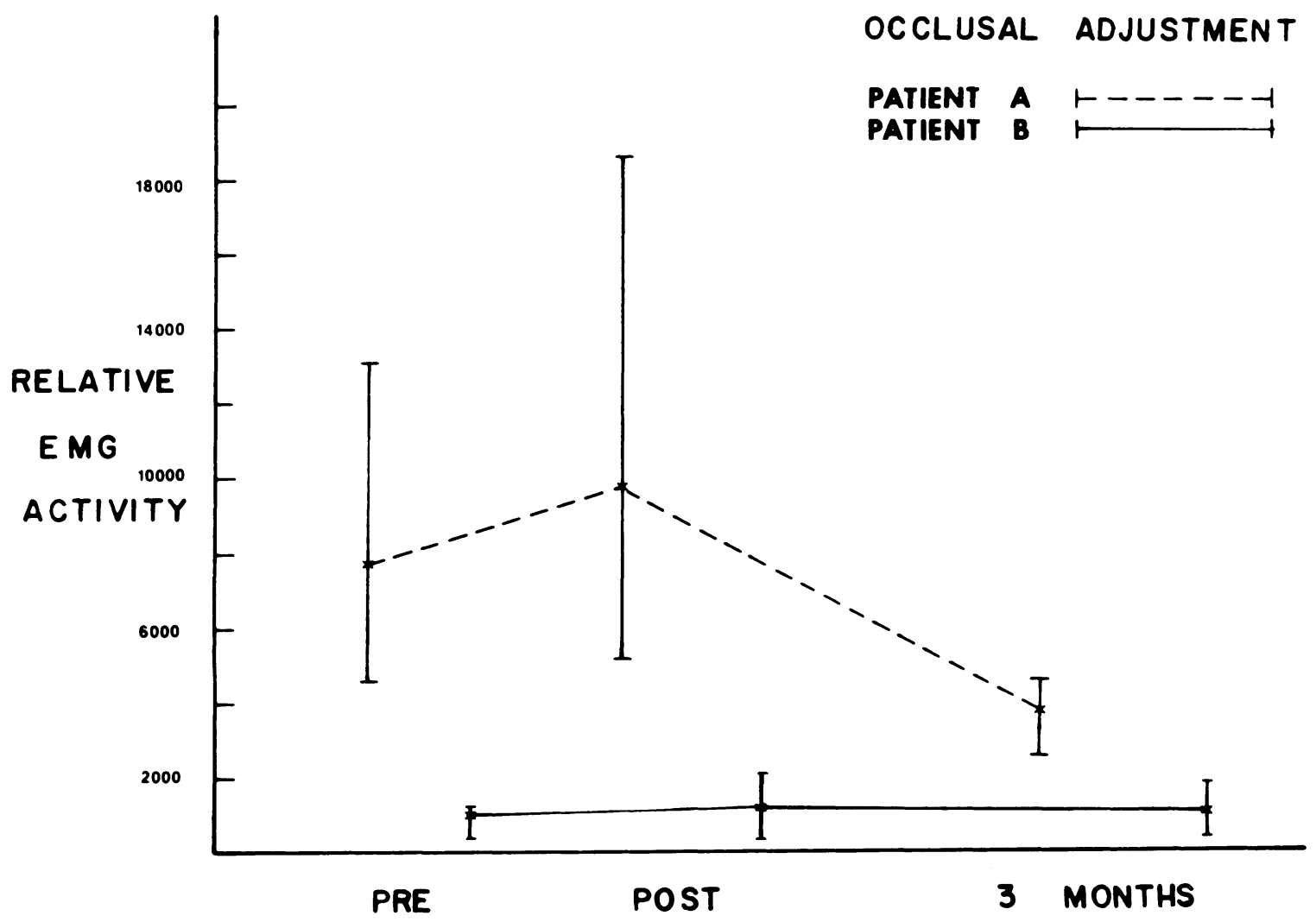

FIgURE 1. The EMG activity of subjects $A$ and B in the Occlusal Adjustment Group. The horizontal axis illustrates the recording period and the vertical axis EMG units. These are relative only to the individual patient and to the threshold level for that patient. Vertical bracket lines represent the range of recorded activity for the sessions. 


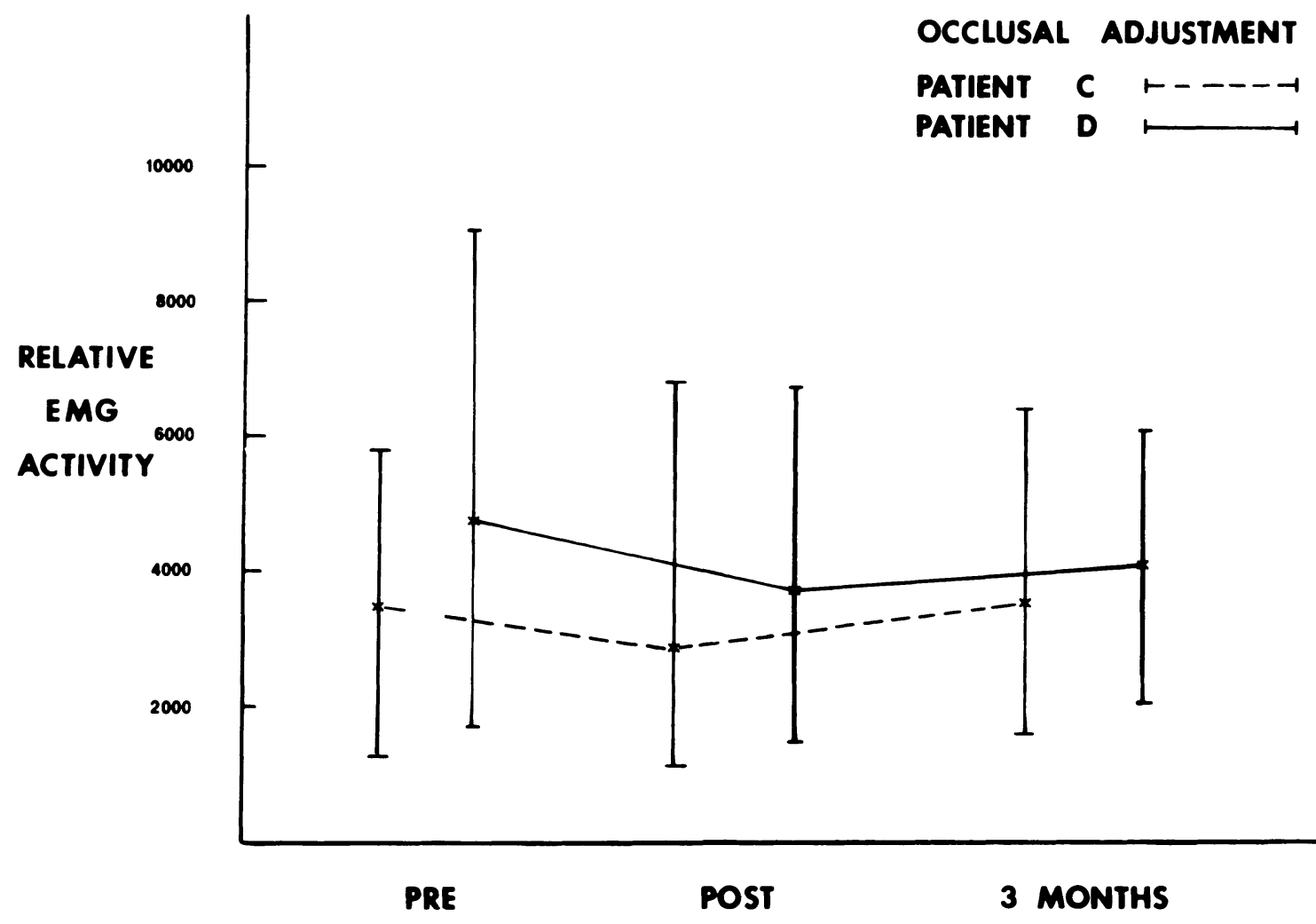

Figure 2. The EMG activity of subjects $C$ and $D$ in the Occlusal Adjustment Group.

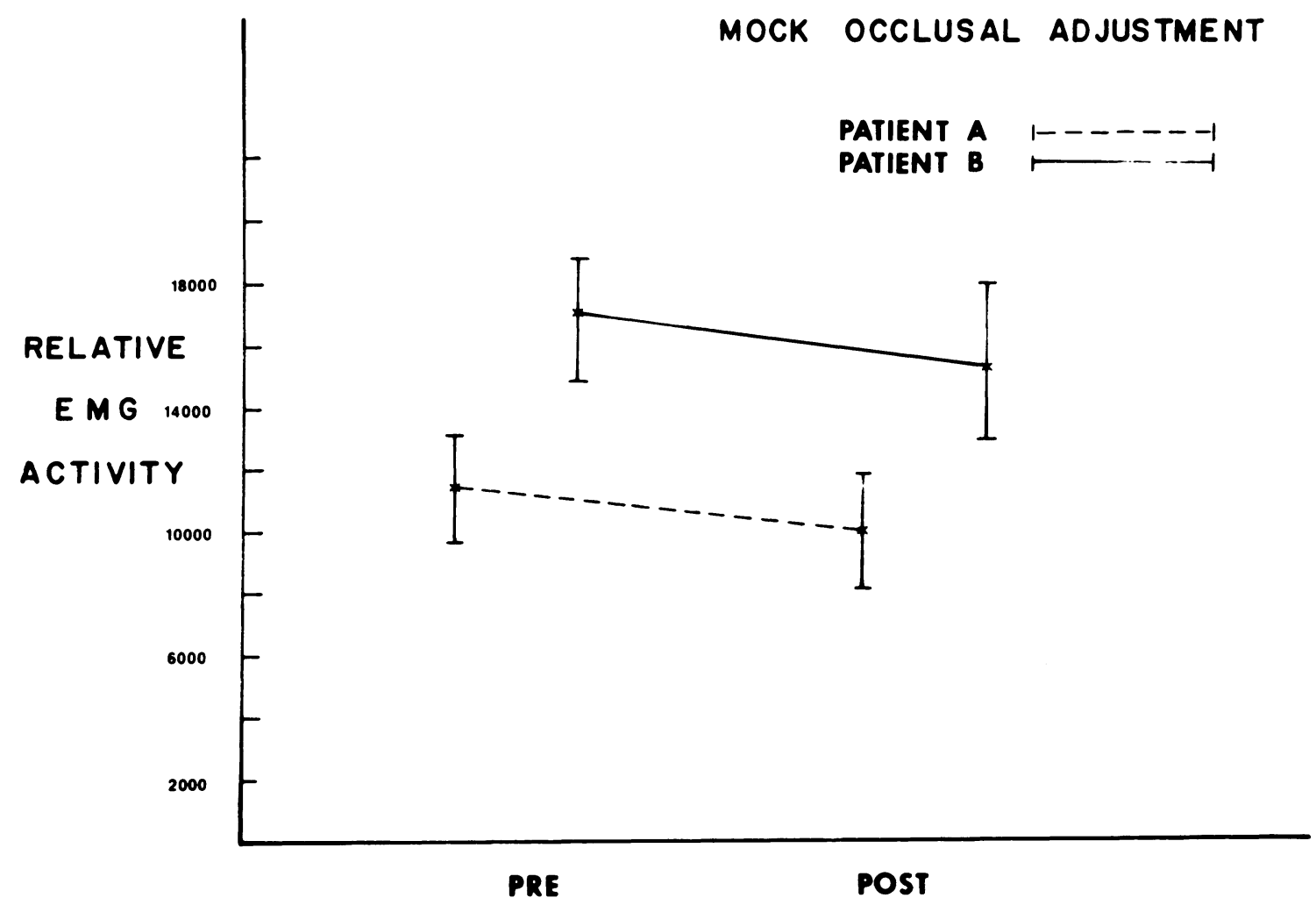

FIGURE 3. The EMG activity of subjects $A$ and $B$ of the Mock Occlusal Adjustment Group. This is representative of all subjects in that group.

recording sessions would be desirable to definitively establish average EMG levels.

A major problem confronting night time studies is that at present there is no method of distinguishing between eccentric bruxism, clenching and unrelated muscle activity, and as the etiologies of these entities may differ, so may the effects of an occlusal adjustment or any other form of therapy. However, with the 


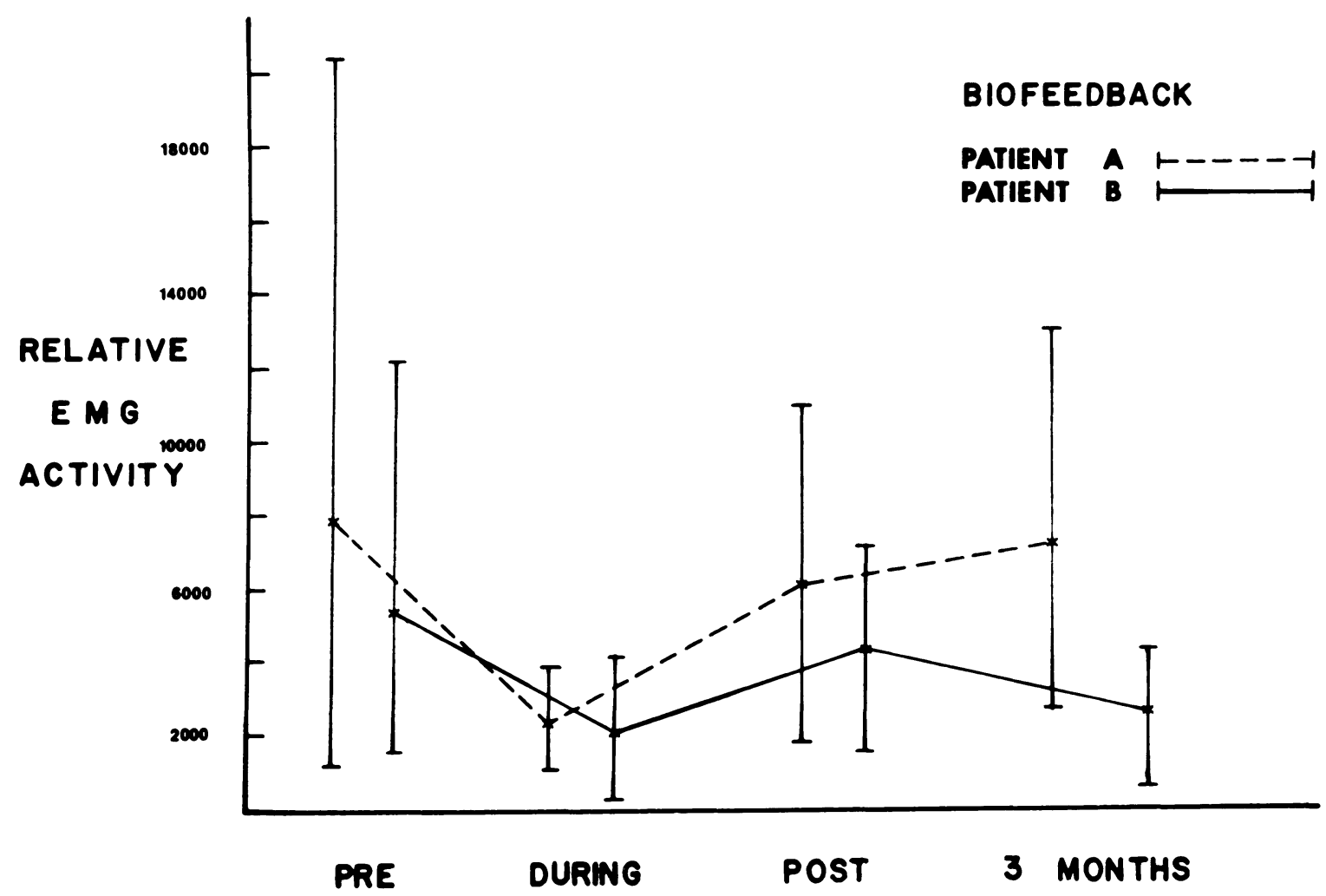

FIgUre 4. The EMG activity of subjects $A$ and $B$ in the Biofeedback group.

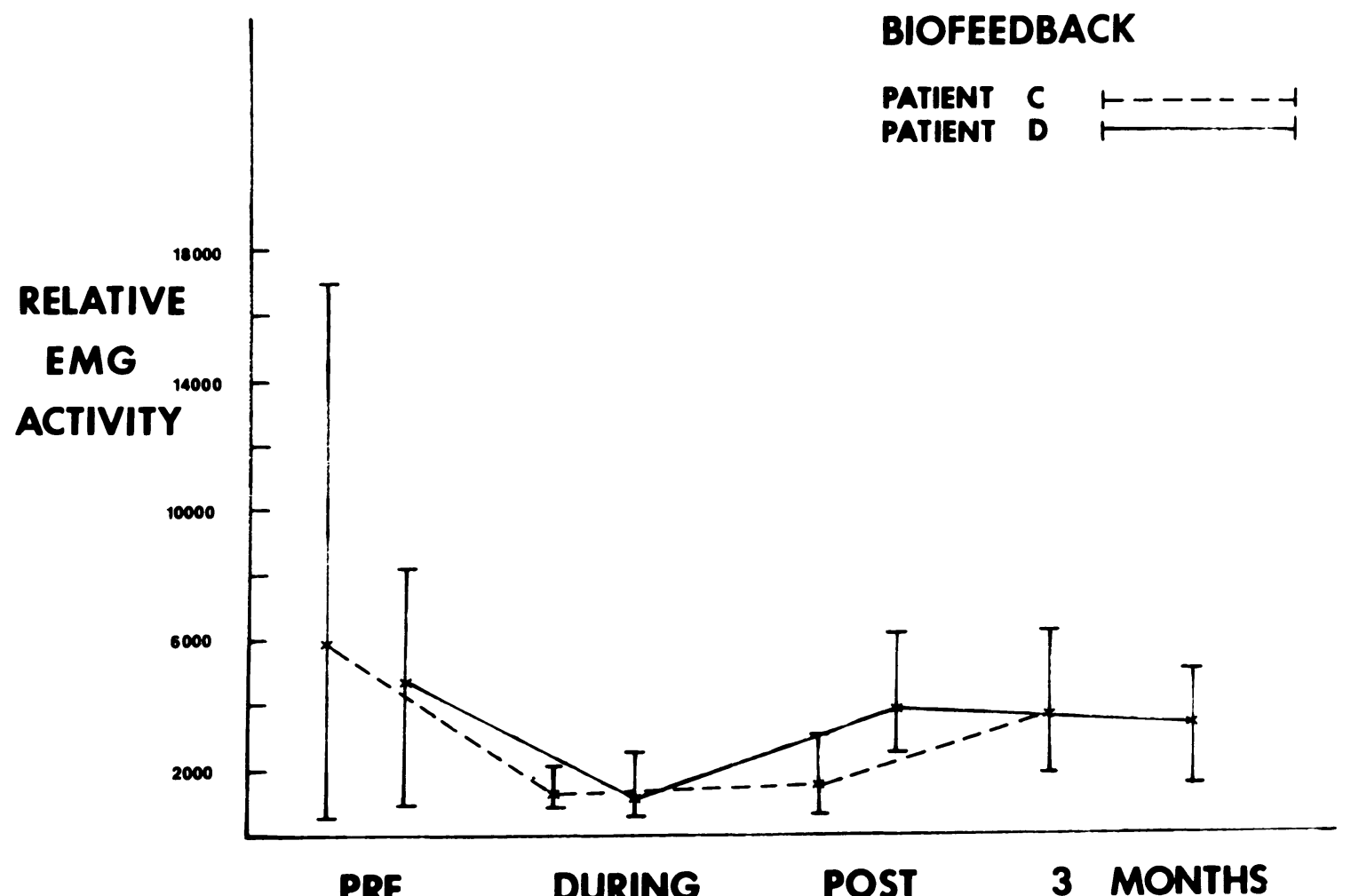

Figure 5. The EMG activity of subjects $C$ and $D$ in the Biofeedback group.

equipment used in this project, the EMG activity recorded must be regarded as from a combination of grinding and clenching.

Indications from this study are that the effects of an occlusal adjustment on nocturnal bruxism are unpre- dictable. This is in contrast to previous daytime EMG recordings and clinical observations. ${ }^{2,3}$ In two subjects there was an increase in EMG activity immediately after the occlusal adjustment. Theoretically this should not be surprising as the alteration in cuspal relation- 


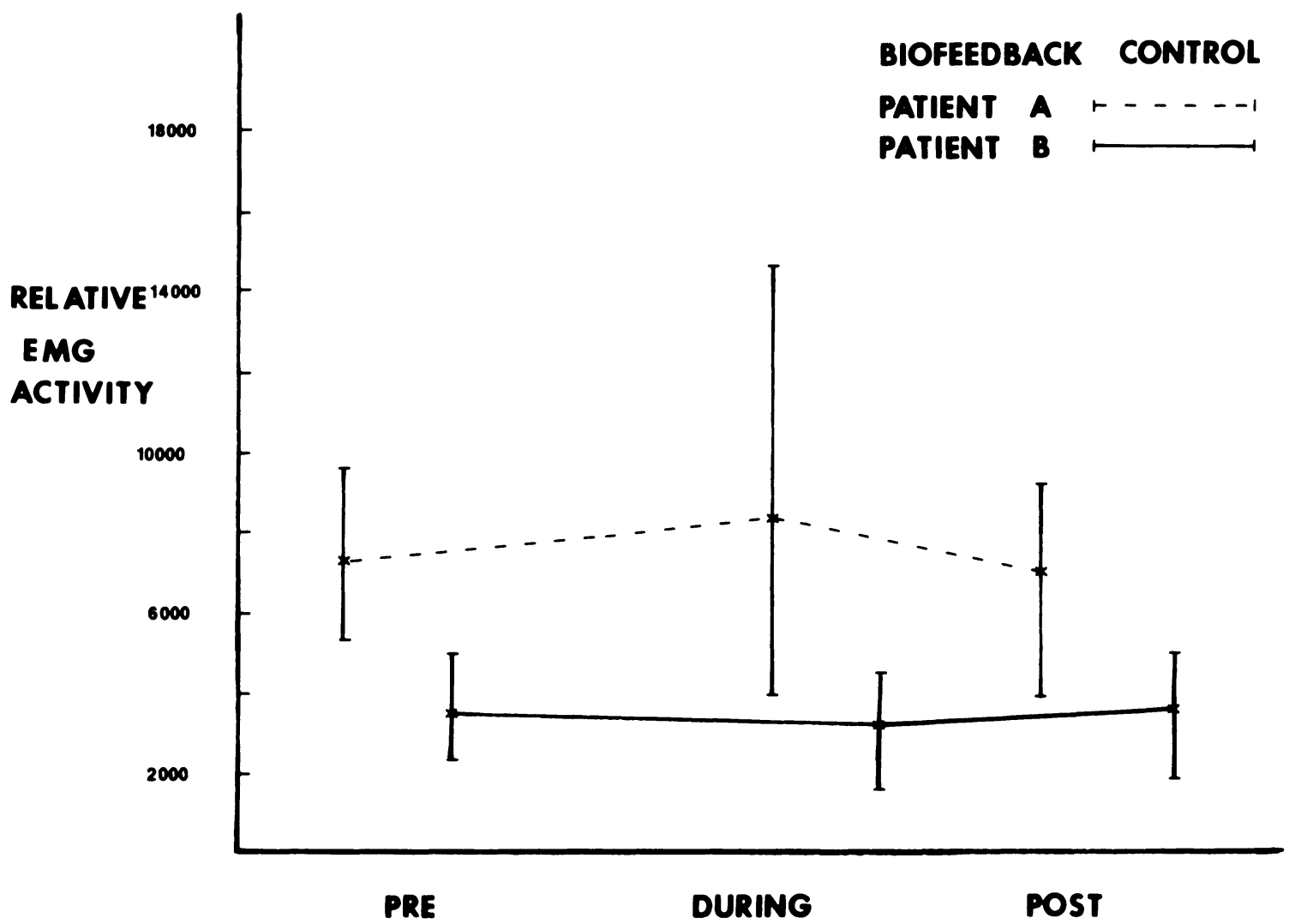

FIGURE 6. The EMG activity of subjects $A$ and $B$ of the Biofeedback control group. This is representative of all subjects in that group.

ships during occlusal therapy may induce patients to "search" for new places and patterns of bruxing or merely "explore" a new "bite". This "doodling" with the altered occlusion could account for the initial increase in EMG activity.

The reduction of nearly 50\% in EMG activity in one subject from the occlusal adjustment group and one subject from the biofeedback group after 3 months may be attributed to the effects of the specific form of therapy, particularly as the range of activity was also reduced. This reduction however, was not predictable at the time of the treatment procedure. On the other hand, the reduction may merely reflect periods of differing stress levels, which again demonstrates the need for long recording periods during each sequence.

Although the mock occlusal adjustment produced very little alteration in the amount of recorded EMG activity, all subjects remarked that the "bite" felt much better and were very pleased with the treatment.

Stress greatly influences bruxing activity ${ }^{1,8}$ and during this study it was obvious that the stress factor could not be controlled in any way. Although subjects claimed that during the recording periods their environment was stable, large variations in activity between nights was observed. No factors responsible for these variations could be isolated from the patients subjective reports. This bears out the importance of a sleepbruxism study because what happens with the subconscious mind during sleep cannot necessarily be corre- lated with a patient's reaction while in the wakeful state.

The reduction in EMG activity while on active biofeedback therapy substantiates earlier results on this treatment modality. ${ }^{4}$ The audible tone relayed instantaneously to the subject was directly related to the intensity and duration of the EMG activity, thus being true feedback of a physiologic phenomenon. The tone did not wake the subject and it can be conjectured that brain wave activity was altered. The return to the initial EMG level on cessation of feedback treatment confirms reports in the medical literature. These studies have shown that for biofeedback to bring about a lasting reduction in monitored activity, a training period is required. ${ }^{9}$ These training periods may be for several months with a wakeful subject who understands biofeedback principles and wishes to control his or her own parameters. With biofeedback and the sleeping subject, different brain wave patterns are present and retention of material applied during sleep is not always predictable. ${ }^{10}$

Biofeedback, as well as reducing the mean muscle activity during treatment seems to control such activity. The range of recorded data between nights appeared to be much less than either before or after the feedback and may explain the relief from "TMJ" symptoms during this therapy. ${ }^{11}$

The continued reduction in EMG activity in one female subject following feedback is difficult to ex- 
plain. It may have been due to the feedback or it may reflect a reduced stress level. The psychological effect of biofeedback with this patient may have been pronounced as she has received other forms of conventional dental treatment unsuccessfully and was very eager to become involved with biofeedback therapy.

The biofeedback control group was an attempt to ascertain that it was actually feedback of EMG activity and not a random tone that caused the reduction in monitored activity. These subjects showed little change in activity while using the "artificial" audible input and it is conjectured that this is because of a lack of association of the audible tone with EMG activity and hence specific brain wave patterns. ${ }^{12}$

The result from the nonbruxist group of subjects demonstrated that two of the subjects did record EMG activity assumed to be bruxism, although the amount was not substantial when compared to the patient groups. The two subjects that did not record any EMG activity above the threshold level can be assumed to be nonbruxers, although they may clench at a level below the threshold used for them in this project. Comparing the data from this group to the other four groups substantiates that the subjects used for this project were bruxers.

During this experiment it was noted that often the first one or two nights of recording in most subjects were higher, possibly an effect of wearing the equipment and partaking in a research program (a new environmental situation). In future experiments the first two nights possibly should not be analyzed along with the succeeding data.

\section{SUMMARY}

Within the limits of this study and with the reported assumption that the recorded EMG activity is related to bruxism, the results of this investigation suggest that the effect of biofeedback in reducing EMG activity is more consistent than an occlusal adjustment, possibly due to differences in the cause of the initial heightened EMG activity in the group of subjects studied.

\section{ACKNOWLEDGMENTS}

The authors wish to thank Dr. Nigel Clarke of Adelaide for the use of his EMG equipment and Mr. Ed Rohan of Ann Arbor for his technical assistance throughout the experiment.

\section{REFERENCES}

1. Solberg, W. K., and Rugh, J. D.: The use of biofeedback devices in the treatment of bruxism. J S C Dent Assoc 40: 852,1972 .

2. Ramfjord, S. P.: Bruxism, a clinical and electromyographic study. J Am Dent Assoc 62: 21, 1961.

3. Dawson, P. E.: Evaluation, Diagnosis and Treatment of Occlusal Problems, ed 1, pp 101-107. Saint Louis, The C. V. Mosby Company, 1974.

4. Kardachi, B. J. R., and Clarke, N. G.: The use of biofeedback to control bruxism. J Periodontol 48: 639, 1977.

5. Rugh, J. D., and Solberg, W. K.: Electromyographic studies of bruxist behaviour before and during treatment. Calif Dent Assoc 3: 56, 1975.

6. Dowdell, L. R., Clarke, N. G., and Kardachi, B. J. R.: Biofeedback: Control of masticatory muscle spasm. Med Biol Eng May, vol. 14, No. 3, 295, 1976.

7. Ramfjord, S. P., and Ash. M. M.: Occlusion, ed 2, pp 271-312. Philadelphia. W. B. Saunders Company, 1971.

8. Yemm, R. B.: Temporomandibular joint-function and dysfunction III. Review No. 7, Munksgaard. Oral Sci Rev $31-53,1975$

9. Budzynski, T. H.: Biofeedback procedures in the clinic. Semin Psychiatry 5: 537, 1973.

10. Simon, C. W.. and Emmons, W. H.: Learning during sleep? Psychol Bull 52: 328. 1955.

11. Clarke. N. G., and Kardachi, B. J. R.: The Treatment of myofacial pain-dysfunction syndrome using the biofeedback principle.J Periodontol 48: 643, 1977.

12. Osward, I.: Sleeping and waking. Physiology and Psychology, pp 35-41. Amsterdam-New York. Elsevier Publishing Co.. 1962. 